\title{
Les défenseurs officieux : une défense sans
} barreaux

The "défenseurs officieux": a Defense without the Bar

\section{Nicolas Derasse}

\section{(2) OpenEdition \\ 1 Journals}

Édition électronique

URL : https://journals.openedition.org/ahrf/11230

DOI : $10.4000 /$ ahrf. 11230

ISSN : 1952-403X

Éditeur :

Armand Colin, Société des études robespierristes

Édition imprimée

Date de publication : 1 décembre 2007

Pagination : 49-67

ISSN : 0003-4436

\section{Référence électronique}

Nicolas Derasse, "Les défenseurs officieux : une défense sans barreaux », Annales historiques de la Révolution française [En ligne], 350 | octobre-décembre 2007, mis en ligne le 01 janvier 2011, consulté le 01 juillet 2021. URL : http://journals.openedition.org/ahrf/11230 ; DOI : https://doi.org/10.4000/ ahrf. 11230 


\section{LES DÉFENSEURS OFFICIEUX : UNE DÉFENSE SANS BARREAUX}

Nicolas DERASSE

Conformément à leur volonté de rapprocher la justice de la Nation, les Constituants autorisent les citoyens à prendre la défense d'autrui devant les tribunaux et à désigner le conseil de leur choix pour les représenter en justice. La liberté de la défense devient ainsi la norme à partir de janvier 1791 et met en avant de nouveaux acteurs de la plaidoirie qu'on nomme les défenseurs officieux. Ces hommes, amenés à remplacer les avocats dont la fonction a été supprimée au début de la Révolution, ne sont astreints à aucune règle professionnelle. Investis de la confiance des parties, ils doivent défendre idéalement l'ami et lui rendre service sans contrepartie financière. Leur parcours, critiqué dès la Révolution, montre que l'Assemblée constituante, malgré ses ambitions, n'est pas parvenue à effacer la règle selon laquelle défendre est un métier réservé aux praticiens du droit, même démunis de leur titre, et délaissé par des particuliers sans bagage juridique. Ainsi, la défense officieuse a surtout été une étape dans l'histoire du barreau, à une période où l'homme de loi, quelle que soit son origine professionnelle, inspire une certaine méfiance et de la crainte aux autorités politiques de la Révolution et du Consulat.

Mots-clés : avocat, liberté de la défense, gratuité, homme de loi, justice populaire, tribunal criminel.

"Heureux celui que la nature et le travail ont destiné à devenir le protecteur de ses semblables et à exercer le plus noble des ministères ! Tels seront les défenseurs officieux "'. En cet hiver 1790, la définition est encore

(1) Dinocheau, 13 décembre 1790, dans J. Mavidal, et L. LAuRENT (dir.), Archives parlementaires $(A P), 1^{e r c}$ série, t. 21 , p. 440 . 
imprécise mais le propos enthousiaste. Il est vrai que les paroles de Dinocheau, député de l'Assemblée constituante, sont prometteuses, rassurantes même, pour l'avenir d'une défense en justice qu'il faut imaginer autrement. À l'heure où il s'exprime, la justice de l'Ancien Régime est déjà largement malmenée. La Nation se l'est déjà appropriée, suivant en cela certaines suggestions émises au sein des cahiers de doléances. La grande loi sur l'organisation judiciaire des 16-24 août 1790 a ouvert la voie à une réforme profonde de l'institution judiciaire. Les débats préliminaires ont permis d'évoquer, entre autres aspects, la situation des avocats et des procureurs. " Milice dangereuse » que ces professionnels, là où d'aucuns souhaiteraient que " la fureur de plaider " ne soit " plus qu'une maladie intermittente $»^{2}$.

Dans un contexte de rejet du corporatisme, les représentants de ces professions savent que leurs heures sont comptées. Pour un barreau que la crise n'a pas épargné $e^{3}$ le coup de grâce survient avec le décret des 2-11 septembre 1790 prévoyant, en son article 10, que " les hommes de loi, ci-devant appelés avocats, ne devant former ni ordre, ni corporation, n'auront aucun costume particulier dans leurs fonctions $»^{4}$. Le temps où les stagiaires servaient de «meubles de décoration » et devaient " redoubler de courbettes et d'intrigues auprès des vieillards » de l'Ordre ${ }^{5}$ est désormais révolu. La suppression du barreau ne laisse pas la défense en justice orpheline, puisque les avoués, amenés à remplacer les défunts procureurs, sont institués par la loi des 29 janvier-20 mars 1791. À ces nouveaux auxiliaires qui pourront aussi provenir de l'ancien barreau, la Constituante octroie le monopole de la postulation et la faculté de plaider.

$\mathrm{Ce}$ dernier texte est trompeur. Par les faveurs qu'il réserve aux juristes de l'Ancien Régime, il masque l'intérêt porté au principe de la liberté de la défense depuis le début de la Révolution française. Dans la lignée des droits individuels consacrés par la Déclaration du 26 août 1789, les députés appliquent ce principe au domaine de l'assistance en justice, estimant que la défense est un droit naturel " fondé sur les premiers principes de la raison et de la justice $»^{h}$. C'est dans cet esprit que naissent les défenseurs officieux, parfaite traduction d'une volonté politique de confier à chaque citoyen le droit de défendre lui-même sa propre cause ou de s'en remettre au défenseur de son choix, homme de loi ou plus simplement parent ou

(2) Chabroud, séance du 30 mars 1790, id., t. XII, p. 444.

(3) Voir Hervé Leuwers, L'invention du barreau français, 1660-1830, Paris, Ed. EHESS, 2006, p. 231 sq.

(4) DUVERGIER, Collection complète des Lois, décrets, ordonnance, Paris, 1834-1949, t. 1, p. 355.

(5) BRISSOT, "Un indépendant à l'Ordre des avocats ", dans Bibliothèque philosophique du législateur, du politique, du jurisconsulte, Berlin, 1782-1785, t. 6, p. 395.

(6) Robespierre, séance du 14 décembre 1790, $A P$, t. 21 , p. 466. 
ami’. Dans le seul but « de donner à la liberté de la défense une plus grande latitude », tout individu doit désormais disposer du "droit de défendre un autre citoyen » devant les juridictions civiles et pénales". S'il s'engage dans cette voie, la loi le désignera comme un défenseur officieux, qui accepte de rendre service - au sens de l'officium -, et qui accomplit un devoir civique, voire un «patronage » sans contrepartie financière, conformément au principe de gratuité ${ }^{y}$. A cette liberté s'oppose le corporatisme. Car c'est bien à une défense sans barreaux, dénuée des contraintes professionnelles dans lesquelles le barreau enfermait ses membres, qu'aspirent les Constituants. Ils la prédisent alors même qu'ils viennent de supprimer l'Ordre des avocats et qu'ils s'apprêtent à faire disparaître les procureurs. Mais les députés restent prudents en laissant la porte de la défense en justice ouverte à ces auxiliaires sans statut. Ces conseils, qualifiés d'hommes de loi, peuvent toujours assister les parties, de manière orale ou par écrit. La libéralisation de l'accès à la défense coïncide avec la faculté offerte à tout accusé, depuis la loi des 8 octobre-3 novembre 1789, « de se choisir un ou plusieurs conseils avec lesquels il pourra conférer librement ${ }^{10}$. L'annonce d'une nouvelle ère pour la plaidoirie pénale " ouvre des perspectives intéressantes pour les représentants de la défense, notamment sur le plan criminel. Linstant est donc bien choisi pour faire cohabiter, au cœur de la défense, citoyens et hommes de loi.

La défense libre n'a été évoquée, pour l'essentiel, qu'au travers des études portant sur l'histoire du barreau ${ }^{12}$ ou, plus généralement, de la défense ${ }^{13}$. Dans ces travaux, c'est surtout la place des hommes de loi durant

(7) Le rôle joué par la famille et les amis est déjà important durant l'Ancien Régime, notamment dans le cadre du procès criminel. Ce relais permet « d'atténucr fortement les risques de l'absence du conseil lors de l'interrogatoire ", A. ASTAING, Droits et garanties de l'accusé dans le procès criminel d'Ancien Régime $\left(X V T^{\prime}-X^{\prime} V I H^{\prime}\right.$ s.) : audace et pusillanimité de la doctrine pénale française, Aix-en-Provence, Presses Universitaires d'Aix-Marseille, 1999, p. 93.

(8) Dinochcau, séance du 13 décembre $1790, A P, 1^{\text {tru }}$ série, t. 21 , p. 440.

(9) C'est la loi des 29 janvier-20 mars 1791 qui consacre la défense officieuse, en offrant aux parties " le droit [...] d'employer le ministère d'un défenseur officieux pour leur défense, soit verbale, soit par écrit "(art. 3), Duvergier, op. cit., t. 2, p. 184. Un second texte du 15 juin 1791 prévoit que « tout citoyen pourra exercer les fonctions de défenseur officieux $"($ art. 26), id., t. 3, p. 41.

(10) Art. 10, id., t. 1, p. 49

(11) Voir Jean-Pierre ROYER, « Parole d'avocat... Remarques sur la plaidoirie pénale, de la fin de l'Ancien Régime à la Révolution ", Droits, n 17,1993 , p. 99-112.

(12) La bibliographie étant particulièrement riche, on orientera le lecteur vers les ouvrages suivants : Hervé LEUWERS, op. cit.; Ugo BELLAGAMBA, Les avocats à Marseille : praticiens du droit et acteurs politiques (XVIIr et XIX's.), Aix-en-Provence, 2001 ; Lenard R. BERLANSTEIN, The baristers of Toulouse in Eighteenth Centuny (1740-1 79.3), Baltimore \& Londres, 1975 ; Michael P. Fitzsimmons, The Parisian Order of Bamisters and the French Revolution. Cambridge \& Londres, 1987 ; Jean-Louis GAZZANIGi (dir.), Histoire des avocats et du barreau de Toulouse du XVIII s. a nos jours, Toulouse, 1992, et, du même, "Les avocats pendant la période révolutionnaire ", dans Robert BADINTER (dir.), Une autre Justice, Paris, 1989, p. 363380. Enfin, Isser Wol.xM, "The fall and Resurrection of the Civil Bar, 1789-1820s ", French Historical Studies, 1987, vol. XV, p. 241-262.

(13) Jean-Louis HalPÉrin, « Droit de défense et droits des défenseurs en France de 1789 a 1914 ", dans L'assistance dans la résolution des conflits, Bruxelles, Recuejls de la société Jean Bodin, t. LXIV, 1997, p. $99-122$. 
l'époque révolutionnaire qui est mise en avant, et la défense officieuse, en tant qu'institution, apparaît au second plan. Une étude spécifique a néanmoins été soutenue sur la question ${ }^{14}$. Elle permet, au même titre que les différentes recherches déjà menées, de cerner les contours de cette fonction créée par les Constituants. Dans ce cadre, les archives judiciaires constituent une source essentielle, notamment pour dévoiler l'identité et l'activité des défenseurs officieux. Partant de l'idée selon laquelle le législateur voyait surtout dans cette fonction un service d'ami, celui-ci avait beaucoup plus de chance de se réaliser devant les juridictions pénales, plus accessibles aux non-juristes que les audiences civiles. Les archives des tribunaux criminels mettent par ailleurs en évidence le nouveau poids de la défense auprès des accusés, ce qui, répétons-le, était une nouveauté en 1789. La défense officieuse pouvait donc trouver dans le procès criminel un terrain propice à son épanouissement ${ }^{15}$.

Afin de prendre la mesure de cette institution, il faut nécessairement dépasser le cadre chronologique de la décennie révolutionnaire. En effet, si la défense officieuse est née en 1790, la dénomination subsiste dans les registres des cours de justice criminelle jusqu'en 1810. Toutefois, la loi du 22 ventôse an XII (13 mars 1804), qui rétablit le titre d'avocat, lui assène un coup décisif en annonçant sa prochaine disparition ${ }^{16}$. Sur ces quatorze années, il est possible de se pencher sur l'évolution de cette institution à partir des critères posés initialement par les Constituants, c'est-à-dire la liberté de la fonction et l'absence de réglementation. En 1790, les députés prennent le risque d'ouvrir la défense à des citoyens sans qualification tout en ne fermant pas l'accès aux professionnels qui, jusqu'alors, assuraient l'assistance des justiciables. Cette audace s'est-elle avérée payante pour la défense ou au contraire, s'est-elle montrée pénalisante pour ce milieu tout autant que pour les parties ? Létude de la composition de la défense officieuse et du comportement de ses acteurs offre des éléments de réponse. La liberté de la défense a ainsi favorisé un état de confusion permanent qui, à défaut d'avoir bénéficié aux amateurs, a permis aux hommes de loi de continuer à exercer leur profession dans l'attente du rétablissement de leur titre.

(14) Nicolas DERASSE, La défense dans le procès criminel sous la Révolution et le Premier Limpire (1789-1810) : les mutations d'une fonction et d'une procédure, th. droit, Lille 2, 1998.

(15) Pour cette ćtude. nous nous sommes appuyés sur les dépouillements effectués dans les archives judiciaires des départements du Nord, de la Vienne et de l'Hérault et réalisés dans le cadre de notre thèse, supra.

(16) Titre III, art. 22 : "Les individus exerçant, au moment de la publication de la présente loi, les fonctions de défenseur officieux près les tribunaux, les continueront provisoirement, sauf l'exécution des règlements de discipline, jusqu'à l'époque fixée pour remplir les conditions qui leur sont imposées; après lequel temps ils seront tenus de justifier de leur accomplissement, ou de discontinuer l'exercice de leur profession ", DUVERGIER, op. cit., t. 14, p. 333. 


\section{Un désordre programmé}

En optant pour le principe de la liberté de la défense, l'Assemblée nationale reste fidèle aux idéaux exprimés en 1789. Rapprocher la justice de la Nation, c'est permettre aux citoyens d'y paraître autrement qu'en qualité de justiciable. Aussi convient-il d'encourager les initiatives de ceux qui veulent assister leurs proches, en les rassurant sur l'absence de réglementation de la défense officieuse. Cet attrait de la fonction ne tarde pas à dévoiler des contreparties qui fragilisent l'institution et pénalisent lourdement les parties ainsi que la marche de la justice.

\section{Le libre accès à la défense}

Il faut mettre au crédit des Constituants d'avoir été prudents dans l'affirmation du principe de la liberté de la défense, ce qui n'a pas été le cas des Conventionnels. Ces attitudes divergentes indiquent qu'il y a bien deux temps dans l'évolution de ce principe. Les débats de décembre 1790 marquent une première étape. Si les citoyens sont en effet admis à exercer la défense officieuse, ils subissent la concurrence des hommes de loi autorisés à poursuivre leur activité de conseil. La liberté est donc proclamée mais elle est tempérée par les attributions que le législateur réserve aux auxiliaires de la justice. Un peu moins de trois années conduisent à la seconde étape, marquée par l'adoption de la loi du 3 brumaire an II (24 octobre 1793) qui supprime les avoués. Cette profession discréditée et guettée par la pénurie préserve trop d'avantages pour des hommes de loi que les députés de la Convention nationale regardent avec méfiance. Par ce texte, ils annihilent les faveurs réservées aux juristes de l'Ancien Régime d'intégrer la profession d'avoué, ce dont les citoyens ordinaires, en théorie, étaient privés ${ }^{17}$. En remplacement des avoués, ils instituent les fondés de pouvoir qui peuvent être choisis, comme les défenseurs officieux, dans la population. Le décret marque l'aboutissement du "processus de déprofessionnalisation " ${ }^{1 /}$ de la défense en justice, désormais entièrement ouverte aux hommes du peuple. Il est l'expression, dans un contexte qui lui est propice, d'une liberté totale de la défense.

En 1791, les autorités n'imposent pas de conditions d'accès à la défense officieuse. Celle-ci est libérée des contraintes que l'Ordre des avocats faisait peser sur ses membres, notamment sur le plan disciplinaire. Aussi chaque citoyen est-il indépendant dans sa démarche, qu'il inter-

(17) En 1792, Adrien Duport, alors ministre de la Justice, avait été l'un des premiers à commenter cette interdiction, indiquant que certains citoyens, venus s'acquitter du droit de patente pesant sur les avoués, étaient parvenus à intégrer cette profession, Gazette des tribunaux, Paris, 1791-1796, t. 5, p. 268 sq.

(18) Jean-Louis HALPÉRIN, " Droit de défense et droits des uéfenseurs en France », op. cit., p. 101. 
vienne pour le compte d'un proche, à la demande d'un client ou à celle du juge. Cette diversité conduit les défenseurs à vaquer "à cet office isolément, comme simples mandataires de leurs clients, sans aucun lien de confraternité qui les unît entre eux et sans aucuns droits de discipline les uns à l'égard des autres ${ }^{19}$. La liberté d'accès à la fonction chasse par ailleurs tout critère de capacité et de moralité. Sur ces aspects, Dinocheau avait voulu être rassurant en indiquant que les parties placeraient nécessairement leur confiance dans un conseil honnête. En résumé, la Constituante ne veut pas faire du défenseur officieux un professionnel, ce qu'il n'est pas légalement puisqu'il est dispensé de la patente exigée, depuis le décret d'Allarde de 1791, de tous ceux qui exercent un négoce, une profession ou un métier"

Lexercice de la défense officieuse offre aussi une grande marge de manouvre. Avant de remplir son ministère, chaque citoyen doit simplement prêter serment de s'exprimer «avec décence et modération " et, en matière criminelle, de n'employer que la vérité dans sa défense. Au titre de la police de l'auditoire, les magistrats peuvent juger utile de rappeler à l'odre le défenseur qui s'éloigne du respect dû à la justice, mais ne sont pas autorisés à écarter le récalcitrant de la défense officieuse. La liberté de parole est ainsi largement consacrée au bénéfice de chaque individu. Rapprochant la défense officicuse du service d'ami, les Constituants pensent que le geste doit être désintéressé. Cette générosité est déjà de mise au sein des "bureaux de jurisprudence charitable " créés en 1790 et chargés « d'examiner les affaires des pauvres qui s'y présenteront ${ }^{21}$. Elle s'observe également lors de la désignation d'office d'un conseil en matière criminelle, admise pour tout accusé, y compris l'indigent. Au-delà des principes, les députés sont conscients des réalités et de la souplesse dont il faudra faire montre à l'égard de ceux qui feront de la défense officieuse leur métier. À tout le moins, « la défense, soumise à la concurrence », aura le grand mérite d'être « moins coûteuse $»^{22}$.

(19) DupIN, Réquisitoires, plaidoyers et discours de rentrée, Paris, 1836-1842, 1. 1, p. 184. On ajoutera que "si le plaideur assisté était présent à l'audience, le défenseur officieux était dispensé de donner la preuve du mandat; dans le cas contraire, il devait faire viser par le président du tribunal le pouvoir accordé par son client ", Jean-Louis HALPÉRIN, " Droit de défense et droit des défenseurs en France ", op. cit., p. 108.

(20) Hervé LeUWERS, op. cit., p. 246.

(21) Loi des 16-24 aoút 1790, titre X, art. 8, DUVERGIFR, op. cit., t. 1, p. 326. Lassistance au profit des plus démunis ne naît pas avec la Révolution française. "Le principe que les hommes de loi devaient [...] prêter gratuitement concours [aux pauvres] apparaît dans la plupart des chartes du midi et quelques coutumes du XIII' siècle, le Livre de Jostice et Plet ou Beaumanoir, bien avant que le règlement de novembre 1364 pour les Requêtes du Palais n'impose aux avocats, procureurs et gens de loi l'obligation de plaider et de faire tous les actes pour Dieu quand il s’agit des pauvres $[. ..] \approx$, B. SCHNAPPER, « De la charité à la solidarité, l'assistance judiciaire française 1851-1972 ", dans Voies nowvelles en hisfoire du droit, Paris, 1991, p. $437 \mathrm{sq}$.

(22) Id., p. 441. 
C'est en pleine conscience de ces libertés que les premiers défenseurs officieux s'avancent à la barre des juridictions. La mise en place de la réforme ne suscite pas beaucoup d'enthousiasme. On décèle parfois une certaine attente, mais aussi de la curiosité chez ceux qui découvrent les prétoires. Ici, c'est un "maitre de comptes » qui apporte son soutien à un ami accusé auprès duquel il a servi durant sept années ${ }^{23}$. Ailleurs, c'est un beau-frère et un neveu qui assistent leurs proches traduits devant une juridiction criminelle ${ }^{24}$. Le service offert par ces citoyens reste éphémère, ce qui est conforme à l'esprit de la réforme. Toutefois, certains perçoivent dans cette fonction libre la possibilité d'exercer momentanément une autre activitée $e^{25}$ alors que d'autres, tel l'imprimeur douaisien Descamps, y voient une vraie chance de reconversion ${ }^{26}$. Dans l'ensemble, la faible participation des non-juristes durant ces premic̀res années trouve son explication dans le poids des traditions judiciaires et dans la confiance que les juges, ainsi que les parties, placent dans les auxiliaires de justice de l'Ancien Régime. Les magistrats ne ferment pourtant pas la porte de la défense officieuse à ceux qui ne disposent d'aucun bagage juridique. D'ailleurs, il leur arrive de soutenir des amateurs qui, en tout état de cause, ont fait preuve de sérieux comme conseil des accusés.

D'un autre côté, on comprend aussi que la défense officicuse est un phénomène conjoncturel. Elle s'épanouit lorsque les circonstances lui sont favorables, en particulier durant la Terreur. À cette époque, les offensives portées contre les hommes de loi, dans un climat de suspicion, font le jeu des citoyens, des patriotes surtout. Par civisme, les hommes du peuple s'investissent plus facilement tant que la cause n'est pas suspecte. Leurs démarches sont surtout encouragées par les accusés qui font appel, avec succès, à lá générosité de ceux qui considèrent la défense en justice comme un devoir. Apparaissent alors au prétoire là un aubergiste, ici un directeur de pensionnat, ailleurs un « ancien chartreux marié ${ }^{27}$ ou bien encore ce professeur de philosophic au collège de Poitiers, nommé Sabourin. Très actif en 1793 à la barre du tribunal criminel de la Vienne, il est de ceux qui connaissent une ascension fulgurante, favorisée par l'estime que lui porte la société populaire locale, voyant en lui un modèle de patriotisme ${ }^{28}$. La carrière de ces conseils reste pourtant provisoire. Avec le Directoire,

(23) AD Hérault, L6322, 1790.

(24) AD Nord, L10816, L10841, 1792.

(25) Tel est le cas du citoyen Lauwreens, traducteur de flamand devant le tribunal criminel du Nord, venu assister plusieurs accusés flamands jugés en 1792, AD Nord, L10797, LI0824.

(26) J.-F. Descamps imprimait notamment tous les documents officicls du tribunal criminel du Nord. On le voit plaider très activement entre 1792 et 1794 . En 1794, ce conscil sera jugé et condamné à mort par le tribunal révolutionnaire de Paris.

(27) P. CATAl AN, La justice révolutionnaire à Montpellier et dans le dépantement de l'Hérault en 1793 et 1794, Montpellier, 1902, p. 14.

(28) AD Vienne, L suppl. 394. Ce parcours s’interrompra brusquement l'année suivante. 
les initiatives des proches s'estompent, même si quelques hommes, tels ces pères qui prennent la parole pour leur fils ${ }^{21}$, trouvent commode et économique d'intervenir. Certains soutiens de la Terreur sont encore capables de manouvrer habilement pour plaider une poignée de dossiers, comme ce Charles-Pierre Fradin, ci-devant « agent national du district de Poitiers»; après la chute de Robespierre, ses ennemis le présenteront comme le " provocateur et l'agent principal de tous les assassinats de ses concitoyens commis sur les échafauds [...] Depuis le 9 thermidor, il a cherché à ménager tous les partis et dans les circonstances importantes, il s'est toujours prononcé pour les terroristes $»^{30}$.

Le Consulat n'offre généralement pas de chance à de tels parcours. Mais il est prêt en revanche à favoriser les citoyens qui font preuve de séricux et de détermination. Même si ces derniers demeurent très minoritaires, on les voit fréquenter les audiences à quatre ou cinq occasions, là où l'ami n'est présent que sur une seule affaire. Leur situation atteste que le libre accès à la défense n'est pas une utopie, si tant est que les magistrats soutiennent ces initiatives personnelles. Tous n'ont pas eu cette attitude mais ceux qui l'ont recherchée, y compris les accusés, n'ont semble-t-il pas eu à le regretter. Si l'on sort du simple service amical, qui n'offre aucune perspective d'avenir, la défense officieuse peut ouvrir des horizons intéressants, particulièrement lorsque se profile le rétablissement des avoués et du barreau. Seuls quelques rares conseils parviennent à saisir ces ouvertures, comme l'« étudiant en droit " Charles Duquesne qui devient avoué en l'an IX après six années de défense officieuse ${ }^{31}$, ou le jacobin de Poitiers Charles-Pierre Fradin qui intègre le barreau ${ }^{32}$. C'est la preuve, certainement, que l'expérience peut effacer le passé.

\section{Les vicissitudes d'une absence de réglementation}

Lorsqu'ils affirment la liberté et la gratuité de la défense, les Constituants n'éliminent pas les risques que l'absence de réglementation peut faire courir à la défense officieuse. Les pratiques néfastes sont pourtant minimisées en 1790. Tout repose sur la confiance des parties et la conscience de ceux qui prendront leur défense, hommes de loi et simples particuliers. Il n'a pas fallu longtemps pour constater les défauts des défenseurs officieux. Chez eux, les comportements contraires à une certaine

(29) AD Nord, L 11766, an V; AD Vienne, L suppl. 419, an VI.

(30) AD Vienne, L 211. Exprimé au lendemain de la chute de Robespierre, ce jugement est à replacer dans un contexte de rejet et de condamnation sans appel des responsables politiques de la période précédente.

(31) AD Nord, 2U1/47, p. 30.

(32) Supra. 
déontologie sont monnaie courante, quelle que soit leur origine professionnelle. Ces attitudes prolongent le sentiment de défiance observé vis-à-vis des auxiliaires de justice sous l'Ancien Régime. Pour de nombreux observateurs, l'avocat mais surtout le procureur, suscitent de la méfiance car on les sait âpres au gain. Dès 1791 , la liberté de la défense rend possible, et permet même d'amplifier ces abus qu'une partie des Constituants redoute, surtout depuis la suppression de l'Ordre des avocats. La défense libre peut permettre à l'individu qui défend sa propre cause de mettre a dans sa plaidoirie trop peu d'ordre [et] beaucoup de discussion " parce qu'il connaît peu "les lois et l'application " ". Elle peut aussi ouvrir la carrière à des « intrigants [qui] ruineront les peuples " que l'on cherche à « soulager » et à qui « la justice coûtera cent fois plus cher qu'à l'ordinaire " ${ }^{34}$. Ces paroles contiennent des avertissements propres à ne pas faire sombrer prématurément la défense officieuse. Mais l'inquiétude est fondée, comme le démontrent les premiers commentaires de la presse sur les procès pénaux. Celle-ci aborde le sujet indirectement, en s'inquiétant du respect accordé au principe de gratuité. Sans surprise, ce dernier ne pèse pas lourd face aux pratiques parfaitement rodées des conseils ${ }^{35}$. On mesure déjà combien il est illusoire d'avoir foi en la gratuité de la défense, sauf à considérer que la défense officieuse ne reste qu'un service d'ami et non un métier exercé principalement par les hommes de loi.

La rétribution du conseil ne disparait pas avec la Révolution française. Elle est même légitime aux yeux de certains, au point d'être recouvrée, si besoin est, par voie d'huissier ${ }^{3 h}$. Afin de faire bonne figure, les juridictions s'efforcent très tôt de rappeler à l'ordre les défenseurs officieux, en indiquant que leurs « frais ne doivent sous aucun prétexte passer en taxe " $"$. À la différence des avoués qui tiennent « de la loi le privilège ou le droit exclusif d'exercer une profession lucrative », la défense officieuse ne constitue point « un objet de commerce, ni une source de richesse sur laquelle on puisse asseoir la base d'une contribution ». Le discours est le même pour l'activité d'homme de loi, non considérée « comme un métier, un négoce, ou une profession qui puisse donner prise à l'impôt ${ }^{3.8}$. En résumé, l'honoraire n'a pas lieu d'être pour celui qui n'est pas taxé. Encore faut-il que tout le monde se conforme à cette règle, à commencer par les juridictions ou les autorités moins crédules qui fixent elles-mêmes le

(33) Obsenvations relatives au nouveau projet sur l'ordre judiciaire, 1790, AN, ADII/44-2.

(34) Martincau, séance du 15 décembre 1790, AP, $1^{\text {tat }}$ série, t. 12, p. 486.

(35) Voir Gazette des tribunaux, op. cit., t. 1, 1791, p. $55 \mathrm{sq}$

(36) AD Nord, L12577, Tribunal criminel du Nord, 1792.

(37) Tribunal de Pontivy, décision du 28 octobre 1792, dans H. Debauve, La justice révolutionnaire dans le Morbihan, Paris, 1961, p. 472.

(38) Gazette des tribunaux, op. cit., t. 5, p. 91 sq. 
montant des sommes versées aux conseils ${ }^{39}$. En 1794, il est réclamé à la Convention « une nouvelle loi assurant une règle uniforme dans ce domaine ", mais les députés laissent se prolonger la diversité d'un tribunal à l'autre ${ }^{41 !}$.

Le critère financier est déterminant dans l'intérêt que le défenseur porte au dossier. De ce point de vue, les affaires civiles sont plus attrayantes pour le conseil que les procédures pénales. Cette situation s'explique en partie par le mécanisme de la désignation d'office en matière criminelle. Pour l'accusé qui en bénéficie, c'est l'assurance de disposer de la gratuité. En revanche, la récompense est souvent minime voire inexistante pour le défenseur officieux, lequel préfère se tourner vers des contentieux plus rémunérateurs. On a là l'explication de la pénurie de défenseurs libres devant les juridictions criminelles là où les tribunaux civils ont été bien garnis du fait d'audiences plus lucratives. Cet écueil est maintes fois dénoncé, notamment sous le Directoire où l'on confie volontiers au ministre de la Justice que « le temps est passé où les citoyens en état de remplir ce ministere interessant, s'empressaient de s'en charger. Pendant près de deux ans, tous, (à l'exception d'un seul) ont constamment refusé de deffendre les accusés qui n'etaient point en etat de payer les honoraires $"{ }^{41}$. La liberté de la défense fait fuir les défenseurs des audiences criminelles pour des raisons qui tiennent surtout à la gratuité. Dès lors, les juridictions pénales fonctionnent avec une poignée de défenseurs officieux qui s'efforcent de combler la pénurie observée sur toute la période. Tous les autres trouvent de bonnes justifications pour refuser le ministère, comme cet ancien du barreau, venu avertir la présidence du tribunal qu'il « ignore parfaitement la forme suivant laquelle les formes criminelles s'instruisent $»^{42}$ ou comme cet autre qui prétexte " un gros rhume " pour excuser son absence à l'audience ${ }^{43}$. D'autres jouent franc jeu en expliquant aux accusés que l'acquittement a un prix que certains sont prêts à payer ${ }^{44}$.

(39) En l'an VII, l'administration centrale du Nord fixe à six francs les émoluments des conseils désignés d'office aux accusés indigents (AD Nord, L123, p. 290, an VII), avant que le ministre de la Justice n'annule cette décision, en rappclant que " les fonctions de défenseurs officieux sont de leur nature gratuites ", AD Nord, 2U1.2, lettre du 11 vendémiaire an VIII.

(40) Robert ALLEN, Les tribunaux criminels sous la Révolution et l'Empire 1792-1811, Rennes, PUR, 2005, p. 100.

(41) Propos rapportés par David Moyaux, Naissance de la justice pénale contemporaine : la juridiction criminelle et le jury - L'exemple du Nord (1792-1812), th. droit, Lille 2, 2000, p. 101 (souligné dans le texte).

(42) Arch. de l'État (Mons), tribunal criminel de Jemappes, reg. 54, p. 89, 12 fl. an III.

(43) Robert AlLEN, op. cit., p. 99.

(44) « Dans des moments comme celui-ci - écrit le citoyen Théry, poursuivi pour vol devant le Tribunal criminel du Nord - il ne faut pas regarder à l'arkent. J'ai pris un bon défenseur qui promet de nous acquitter moyennant quarante écus ", AD Nord, 2 U1 139, souligné dans le texte. Lacquittement fut obtenu par le conseil. 
À l'audience, il n'est pas rare d'entendre des défenseurs tenir des propos immodérés, voire injurieux, en s'adressant notamment aux témoins. Les plus téméraires parviennent à les tourner en ridicule pour mieux les discréditer aux yeux du jury. On en voit même " outrager les mœurs publiques " et « offenser la morale » en provoquant, à la grande satisfaction du public, « des combats scandalcux en pleine audience publique entre le ministère public et eux ${ }^{45}$. Certaines scènes sont inclassables, tant l'audace des défenseurs libres paraît illimitée. Lexemple de l'ancien avoué parisien Baret est un millésime. À son client, il indique qu'il connaît un ami intime du rapporteur et que celui-ci lui a promis un soutien dans le dossier, moyennant « quelques paquets de fruits secs et un panier de vin $»^{46}$.

Ces comportements pénalisent surtout les justiciables, abandonnés ou mal assistés par des défenseurs sans scrupules qui leur ont été, le plus souvent, désignés d'office. La justice pâtit elle aussi de l'attitude de ces individus. Elle ne trouve d'ailleurs pas systématiquement la parade, si l'on en juge par la fréquence des jugements rendus hors la présence du défenseur officieux ${ }^{47}$. Elle parvient à remédier aux écueils en prenant des initiatives favorables aux accusés. Là, c'est un juge, voire un accusateur public qui se propose d'assurer la défense ${ }^{4 x}$. En d'autres lieux, on renvoie l'affaire à une date ultérieure pour laisser au défenseur la possibilité de se présenter à l'audience. Linstauration d'un " conseil des pauvres ", ou d'un " comité officieux et gratuit de conseil », selon les dénominations retenues, fait aussi partie des réponses. Elle consiste, sans grand succès d'ailleurs, à regrouper auprès d'une juridiction des défenseurs " d'office qui, payés par l'État, pourront $[\ldots]$ assister gratuitement les prisonniers dans tout le cours de la procédure $»^{49}$.

Dès l'origine, les autorités s'inquiètent de ces dérapages. Mais en dépit des plaintes émises par les juges, surtout depuis le Directoire, l'État reste frileux, comme il l'a été depuis 1789. Le contexte politique apporte des explications, en mettant en évidence les divisions. En l'an V, Merlin de Douai, alors ministre de la Justice, essaye de réagir en appelant les magistrats à « réprimer les dialogues déplacés » de certains conseils ${ }^{50}$. Il les incite

(45) Obsenvations des tribunaux criminels sur le projet de code criminel, Paris, an XIII, t. 5, p. 25, tribunal criminel du Nord.

(46) Jean-Louis HAI.PF́RIN, "Avoués et hommes de loi auprès du tribunal de cassation ", Revue de la SIHPA, $\mathrm{n}^{\circ} 1,1984, \mathrm{p} .94$.

(47) Ainsi, dans le département du Rhône, entre 1792 et 1811 , un accusé sur six est jugé sans conseil, avec un taux d'absentéisme du défenscur qui atteint "son maximum sous Napoléon ", Robert ALLEN, op, cit., p. 101.

(48) Telle est l'initiative prise à Paris en 1791 devant les tribunaux criminels provisoires, Gazette des tribunaux, op. cil., t. 1, p. 299 sq.

(49) $l d .$, t. 1, p. 159 sq. Ces initiatives ont souvent été perçues comme des tentatives de reconstitution d'un barreau " of ficieux", cf. H. THOMAS, Le' Thibunal criminel de la Meurthe sous la Révolution (1792-1799), th. droit, Nancy, 1937, p. $142 \mathrm{sq}$.

(50) La France judiciaire, 1876-1877, t. I, p. 480 
d'ailleurs à appliquer avec rigueur les dispositions de son récent Code des délits et des peines, notamment celle permettant de maintenir l'ordre à l'audience, au besoin en interdisant « pour un temps déterminé la défense officieuse $»^{51}$. Le Conseil des Anciens adhère au propos, lui qui estime " que le défaut de règles à l'égard de ceux qui se présentent au nom d'autrui devant les tribunaux, donne lieu à des abus journaliers ${ }^{52}$. «Il y a urgence ", disent même les députés, mais pas au point de sacrifier la défense officieuse. L'État semble comprendre que la source des inconduites tient surtout au défaut de réglementation de la fonction mais la liberté demeure le meilleur rempart contre les excès d'un corporatisme qui peut renaître. Le Consulat hérite ainsi de ces individus sans statut. Contrairement aux assemblées de la Révolution, attachées à leurs principes, il ne peut faire l'économie d'une réforme que réclament les magistrats. Unanimement, ces derniers avancent quelques suggestions que les excès ont rendues nécessaire. Les mots décence, modération, serment, discipline, capacité, honoraires, moralité reviennent inlassablement. Un terme apparaît aussi fréquemment dans le discours, celui de barreau...

\section{L'obstination des hommes de loi}

Pour reprendre la définition qu'en donne la Gazette des tribunaux en 1792, l'homme de loi est celui « qui s'adonne à l'étude des lois, qui les médite et cherche à en saisir l'esprit et le vrai sens : c'est encore, si l'on veut, un homme qui, après avoir pris des grades dans une Faculté de droit, s'est fait recevoir avocat ensuite dans une cour de justice, et a consacré sa vie à l'étude de la jurisprudence et des lois ${ }^{43}$. Ce terme d'homme de loi, apparu au XVIII siècle, est « trompeur ». Lui qui a vocation à s'appliquer à un "groupe social caractérisé par une commune connaissance du droit ", dans lequel se situent des procureurs ou bien encore des notaires, a surtout bénéficié aux avocats ${ }^{54}$. Invités par les Constituants à rejoindre la défense officieuse, les auxiliaires de justice de l'Ancien Régime contribuent amplement à ce que la défense libre ne soit pas un naufrage. Mais ces hommes, de par lcur passé et leurs idées, attisent la méfiance des pouvoirs publics, lesquels leur rendent la tâche parfois difficile.

\section{Des juristes sans robe}

Une minorité d'hommes de loi, jadis membres du barreau, a trouvé place au sein de la nouvelle magistrature, comme l'y invitait la loi des 16-24

(51) Ibid.

(52) AN, ADII, 43-2, séance du 29 ventôsc an VI.

(53) Op. cit., t. 5, p. $91 s q$.

(54) Jean-Louis HaLPÉRIN, “ Haro sur les hommes de loi », Droits, 17, 1993, p. 55. 
août 1790. Pour tous ceux qui n'ont pas été tentés par cette fonction ou par d'autres ${ }^{55}$, la défense officieuse a pu faire office de bouée de sauvetage. Les Constituants ne sont pas unanimes sur cette intrusion des juristes au sein d'une fonction plus proche du service d'ami que d'une véritable profession. D'aucuns redoutent par ce biais le prolongement de la chicane tandis que d'autres cherchent à convaincre l'Assemblée de la nécessité d'admettre les hommes de loi à l'exercice de la défense officieuse. C'est la position pragmatique de ceux qui ont un peu de considération pour la défense en justice. La prudence conduit à ne pas rejeter ces professionnels sur lesquels repose le succès de la réforme. Lavocat est avant tout un homme de parole et la défense officieuse consiste surtout à plaider. Si son statut disparaît, son expérience peut encore être utile au moment où la nouvelle justice se met en place.

On les devine certainement un peu perplexes, ces hommes de loi, lors des premières audiences. Mais ils sont prompts à s'engager dans la défense officieuse au civil comme au pénal. À la façon dont ils se désignent, on décèle une volonté de se différencier des amateurs ${ }^{5 h}$ avec lesquels ils ne veulent avoir en commun que l'activité. La plupart se présentent aux juges comme hommes de loi, mais il n'est pas rare de les voir employer le qualificatif de défenseur officicux alors même qu'on croirait, à tort d'ailleurs, celui-ci réservé au simple citoyen ${ }^{57}$. Comparativement au grand nombre d'avocats et de procureurs en activité à la fin de l'Ancien Régime, les hommes de loi n'arrivent pas en masse devant les nouvelles juridictions. On les sait plus attirés par les procès civils, où la plupart reprennent leurs habitudes, mais certains ont hâte d'être confrontés aux jurés criminels. Ainsi, sur les quatre-vingt-neuf avocats rattachés au Parlement de Flandre en 1789, près d'une vingtaine propose ses services aux accusés jugés à Douai en 1791 et en 1792 par le tribunal de district de la ville ${ }^{5 x}$. Parmi eux, quelques novices, qui ont prêté serment au moment de la suppression de l'Ordre, mais aussi d'anciens confrères plus aguerris à l'exercice de la défense et souvent plus persévérants comme défenseurs officieux. Pour certains, les audiences du début de la Révolution augurent une longue carrière en matière de défense pénale. Il y a forcément des compétences chez ces professionnels, ce que ne démentirait pas François-Joseph

(55) La carrière politique et les emplois administratifs ont été des reconversions fréquentes.

(56) Quelques uns de ces amateurs se présentent comme hommes de loi, ce qui montre que la notion reste floue.

(57) D'un département à l'autre, d'une ville à l'autre, les comportements diffèrent. Ainsi, " l'Almanach de Tours (de l'an V) sépare très nettement les avocats, qualifiés hommes de loi, et les défenscurs officieux, ce qui sous-entend que ces derniers ne sont pas d'anciens avocats " (le groupe des défenseurs officieux « comprend 29 noms recrutés parmi les avoués ou les ex-procurcurs d'Ancien Régime $"$ ), Pascal PLAs, Avocats et barreala dans le ressort de la Cour d'appel de Limoges de la Révolution française à la Seconde guerre mondiale, th. d'histoire, Paris IV, 1997, p. 118.

(58) On ajoutera que sur les 121 dossiers examinés par le Tribunal criminel du Nord en 1792, 111 sont plaidés par des hommes de loi (ces derniers sont au nombre de 16). 
Deberckem, lui qui a défendu plus de neuf cents accusés traduits devant le tribunal criminel du Nord de 1792 à l'an IX. Le parcours de cet ancien avocat est néanmoins atypique. La grande majorité des hommes de loi ne peut se prévaloir en effet d'une telle assiduité, qui plus est au sein d'une même juridiction.

D'un ressort à l'autre, les suffrages se portent aussi sur des praticiens qui n'ont pas fréquenté le barreau. C'est le cas notamment au tribunal criminel du département de la Vienne devant lequel les anciens procureurs, devenus avoués en 1791, sont nombreux à se présenter pour assurer la défense des accusés s". Dans une moindre mesure, le même constat s'applique pour le tribunal criminel de l'Hérault, au moins lors des premières années de la Révolution ${ }^{\text {ntil }}$. Cependant, ces choix sont souvent conjoncturels. En désignant des avoués, magistrats et accusés placent leur confiance en des conseils qui disposent encore d'un statut, ce qui n'est plus le cas des défunts avocats. Cette reconnaissance légale est à même, dans certains départements, de rassurer les parties et les juges. En revanche, la carrière des anciens procureurs connaît un net fléchissement lorsque la profession d'avoué est supprimée en l'an II. Le champ est de nouveau libre, alors, pour les avocats de jadis, surtout au pénal, tandis que les avoués sans titre, postulants avant tout, se sont tournés prioritairement, en tant que fondés de pouvoir, vers les audiences civiles. Au lendemain de la Terreur, l'ancien barreau devient une composante essentielle de la fonction libre. Sa place n'efface pas celle des avoués, mais se renforce au fil des années pour parvenir même, devant certaincs juridictions, à une situation de quasi monopole. Tel est le cas du tribunal criminel du Nord devant lequel les dossiers échoient, au cours du Directoire, à une dizaine d'anciens avocats dont quatre sont particulièrement actifs. En bénéficiant à nouveau d'un statut en l'an VIII, les avoués font un retour remarqué au sein de la défense officieuse, sans faire trop d'ombre toutefois aux hommes de loi passés par le barreau. Ceux-là mettent à profit l'expérience acquise depuis la création des tribunaux criminels pour préserver leur activité dans l'attente du rétablissement de leur titre.

Il est incontestable que la défense officieuse a reposé, en grande partie, sur les hommes de loi ${ }^{\prime \prime \prime}$. Cependant, seule une minorité d'entre eux a

(59) Sur les 21 affaires plaidées en 1792 devant la juridiction, 2 l'ont été par d'anciens avocats. Le reste des dossiers, ou presque, a été confié aux avoués qui, pour la plupart, avaient été procureurs au Présidial de Poitiers.

(60) 9 avoués sont présents en 1792, ainsi que 7 avocats.

(61) Ce constat est confirmé par les études menées sur d'autres départements. On renverra le lecteur aux travaux de L.R., BERLANSTEIN, op. cit. (p. 169) pour le département de la Haute-Garonne : pour l'Yonne, cf. C. BLUM, Histoire du droit pénal dans le départemem de l'Yonne pendant la Révolution française (1792-1799), th. droit. Paris, 1964, p. 97. Dans le département de Ja Meurthe. sur la période 1792-1799, 679 dossiers criminels ont ćté jugés. Tous les accusés ont été défendus par des hommes de loi, à une exception près, voir H. ThOMAS, op. cit., $145 \mathrm{sq}$. La place des avocats est encore essentielle à Marscille, comme l’indique U. Bellagamba, op. cit., p. 239 sq. 
pu vivre de cette activité, tout au moins lorsqu'elle a été exercée devant les juridictions criminelles. Ces praticiens rassurent ceux qui ont à les désigner, les magistrats les premiers, agissant souvent par réflexe corporatiste. La tradition judiciaire est encore bien présente dans les démarches qu'entreprennent les ex-avocats pour combler les écueils nés de la suppression de l'Ordre en 1790. Dans quelques villes, ces hommes de loi s'organisent et tentent de maintenir l'esprit du barreau. Il y a bien sûr ces fameux « avocats du Marais » de Paris qui, sous la houlette de Férey et Bellart, préservent les usages et œuvrent, le moment venu, au rétablissement de leur titre mais l'initiative est présente ailleurs, à Bordeaux notamment, durant l'année $1801^{12}$. Le législateur n'est pas étranger à ces velléités de poursuite des traditions professionnelles. À sa manière, il les encourage lorsqu'il prône, au cours du Directoire, le rétablissement du tableau et des avocats qui le composaient jusqu'en $1790^{63}$.

Plus que les amateurs, les hommes de loi affichent leurs qualités dans l'exercice de la défense officieuse. Au pénal, l'adresse repose en partie sur la faculté de prendre le public à témoin sur les dossiers les plus sensibles. Dans une atmosphère d'effervescence populaire, les magistrats et le juge peuvent céder à la pression, à la plus grande satisfaction d'un public conquis par les arguments de la défense ${ }^{\text {t4 }}$. Le conseil peut parvenir à ce résultat par ruse, mais sans se départir d'un « charme » qui est propre à " subjuguer l'auditoire», voire à « faire couler les pleurs »".5. Il lui faut encore «plaire et persuader ", comme sait le faire l'ancien avocat Fournel devant les nouvelles juridictions de la Révolution ${ }^{\text {th. }}$. Les compétences s'apprécient également au travers des défenses écrites que les hommes de loi continuent de rédiger, comme ils le faisaient avant 1789. Par le biais d' " observations justificatives » et autres requêtes qui rappellent les mémoires judiciaires de l'Ancien Régime ayant contribué à faire naître l'opinion publique $^{67}$, le défenseur officieux argumente et complète la plaidoirie, souvent de manière plus ordonnée qu'à l'oral. Pour les juristes, les deux exercices sont proches, au point que certains mémoires d'audience, rédigés à la première personne du singulier, laissent à penser que la plaidoirie, loin d'être une improvisation, repose sur un support écrit.

(62) Hervé LeuWERS, op. cit., p. 255.

(63) Régnicr et Delzons, Conseil des Anciens, séance du 29 ventôse an VI, AN, ADII, 43-2.

(64) C'est ce que parviennent à réaliser les anciens avocats montpcllićrains Serres et Rech devant le tribunal de district de la ville en 1791 , cf. J. Vtekctr.r, La justice criminelle dans le département de l'Hérault pendant la Révolution (1789-1800), th. Droit, Montpellier, 1925, p. $112 \mathrm{sq}$.

(65) AD Nord, 2U1/13.

(66) Gazette des tribunaux, op. cit., t. 1, p. 197.

(67) Sur ce point, voir Sarah M^ZA, Vies privées, affaires publiques. Les causes célèbres de la France prérévolutionnaire, Paris, Fayard, 1997. 
Les hommes de loi restent des défenseurs officieux assidus et plus à même que les amateurs d'assurer la défense des parties. Pour autant, ils ne sont pas exempts des maux reprochés à une frange de la défense libre. L'avidité, l'absence à l'audience, les propos abusifs ne sont pas l'apanage de citoyens ordinaires. Une partie des hommes de loi entretient la mauvaise image de la défense officieuse. Les autres, en revanche, ouvrent, par leurs qualités, à la bonne marche de l'assistance en justice et contribuent à ce que la défense libre ne sombre pas prématurément. Un tel comportement vaut bien le rétablissement d'un cadre professionnel auquel certains veulent encore croire. Celui-ci viendra d'abord en 1800 , avec le retour des avoués et, parmi eux, des anciens procurcurs en grand nombre, ensuite en 1804, grâce au rétablissement d'un titre d'avocat qui sera réservé en priorité à ceux qui l'ont déjà portétig.

\section{Les obstacles dressés par le pouvoir politique}

Depuis 1789 , l'homme de loi, quelle que soit son origine professionnelle, fait l'objet d'une surveillance particulière. Il n'y a pas que les juges qui inspirent de la méfiance aux pouvoirs publics. En supprimant les fonctions de procureur et d'avocat, les Constituants font entrevoir leur mépris des structures de la défense, coupables de freiner les initiatives personnelles. Celles-ci peuvent enfin s'épanouir grâce au libre accès à l'assistance en justice. Cette garantie est paradoxalement de nature à entretenir l'inquiétude à propos du comportement des conseils. Elle a été obtenue en effet par la suppression de tout ce qui permettait de contrôler les agissements des représentants de la défense. Les hommes sont également redoutés, car ils peuvent contribuer à faire échec à une politique de répression. Ces menaces recommandent donc un contrôle et une vigilance qui sont toujours de mise lors des réformes portant sur la procédure pénale et sur le statut des auxiliaires de justice.

Les hommes de loi justifient ces inquiétudes parce que leur histoire et leur pratique ne se sont pas éteintes avec la Révolution française. Contribuant à la naissance de l'opinion publique au siècle des Lumières, ils sont souvent les porte-parole de la Nation dès 1789 et pour certains d'entre eux, de futures grandes figures politiques. Ecoutés dans et audehors de l'enceinte judiciaire, ils apportent leur expérience là où d'autres font l'apprentissage de la défense en justice. Le pouvoir appréhende chez ces conseils, comme l'indique Champion de Cicé en 1789, la tentation « de faire fléchir les principes à la nécessité de la défense, de dénaturer les circonstances pour atténuer la force des preuves $[\ldots] »^{h 9}$.

(68) Ainsi, sur les 18 praticiens inscrits sur le tableau des avocats près les cours de justice de l'Hérault, dressé en 1806, 9 appartenaient à l'ancien barreau de Montpellier.

(69) $A P, 1^{\text {ser }}$ série, t. 10 , p. 464. 
Lune des grandes offensives portées contre les hommes de loi intervient avec la suppression de l'Ordre des avocats et des communautés de procureurs. Dans l'histoire de la défense officieuse, l'institution du certificat de civisme pour exercer la fonction libre, presque concomitante à la suppression des avoués, est aussi importante. Dans le contexte de la Terreur prochaine, le législateur impose en effet un passcport politique à une certaine catégorie de conseils. Désormais, « nul ne pourra être admis à exercer les fonctions d'avoué, homme de loi ou d'huissier auprès des tribunaux civils et criminels, sans justifier de son civisme par un certificat du conseil général de la commune du lieu de sa résidence, approuvé par le directoire de district et visé par celui du département $\gg^{7 n}$. Ce sésame patriotique, accordé ou refusé sans obligation de motiver, pénalise en premier lieu des hommes de loi souvent critiqués pour leur soutien fréquent à la cause royaliste et que Danton dépeint comme des individus « d'une aristocratie révoltante $»^{7}$.

Face à ces grands animateurs de la défense officieuse, la rigueur est de mise dans l'attribution du document. C'est le cas à Paris où le conseil général de la commune, "investi d'une sorte de pouvoir disciplinaire sur les défenseurs officicux », prend « son rôle très au sérieux » et prévient qu'il va « procéder à l'épuration du barreau $»^{72}$. Par peur de se voir refuser le document, beaucoup se détournent de la défense en justice et deviennent, de ce fait, suspects aux yeux du régime. Ceux qui poursuivent l'activité prennent le risque de ne pas obtenir le certificat et d'être placés en détention pour une durée indéterminée. Quelques piliers de la défense officieuse en ont fait l'amère expérience, à commencer par l'ancien avocat douaisien Desprès, victime de la loi des suspects inspirée par son exconfrère Merlin de Douai ${ }^{73}$. D'autres n'ont pas fait preuve du même courage lorsqu'il s'est agi de justifier un désengagement pour la défense officieuse. « Je ne me suis pas empressé de demander un certificat de civisme - écrit cet ancien du barreau - d'ailleurs, je n'en avois pas besoin. Doué malheureusement d'une mémoire trop ingrate, $\&$ trop timide en même temps pour oser plaider en public \& de vive voix, j'ai été contraint de renoncer entièrement à la profession d'avocat, pour me borner à celle d'homme de loi consultant $»^{74}$.

Aux hommes de loi, on fait payer l'absence de patriotisme, l'appartenance à « l'aristocratic » et le soutien à la cause royaliste. À l'époque où le

(70) Loi des 26-29 janvier 1793, art. 1, DUVERGiler, op. cit., t. 5, p. 127 sq.

(71) Convention nationale, séance du 22 septembre $1792, A P, 1^{\text {tec }}$ séric, t. 52, p. 84.

(72) J. Delom de MÉzeraC, “ Le barreau libre pendant la Révolution ", Revue des Deux Mondes, 1911, t. 1, p. 585.

(73) Incarcéré d'octobre 1793 à janvier 1794, cet homme de loi, très assidu au tribunal criminel, avait d'abord obtenu le certificat de civisme avant de se le voir retiré. 11 devait cette suspicion à l'émigration de deux de ses fils ainsi qu'à ses opinions royalistes.

(74) AD Nord, L10166. 
gouvernement révolutionnaire restreint les droits de la défense accordés aux ennemis de la République, il en est aussi qui sont inquiétés pour leur activité au sein des juridictions d'exception, comme Chauveau-Lagarde qui perçoit comme un « malheureux honneur » le fait " d'être défenseur au tribunal révolutionnaire ${ }^{75}$. Le conseil de la veuve Capet sait à quel point certaines causes peuvent s'avérer néfastes et se terminer, au micux, par un séjour prolongé en prison, comme celui que subit l'homme de loi arrageois Leducq à la suite d'un acquittement ayant déplu au représentant en mission Joseph Le Bon ${ }^{7 t}$. Dans le pire des cas, les défenseurs officicux payent de leur vie leurs convictions politiques et leur activité, à l'image de ces vingt-huit avocats bordelais exécutés durant la Terreur".

Le contrôle politique instauré par les Conventionnels affaiblit un peu plus la défense officieuse. Outre ses faibles effectifs, la fonction libre est divisée du fait des complaisances accordées à certains de ses acteurs, admis à plaider bien qu'étant démunis de certificat de civisme. À ceux-là, on explique, comme le fait Fouquier-Tinville à l'adresse de l'avocat royaliste Lavaux, que le document ne sert qu'à « contenter le peuple ». De plus, " pour défendre des conspirateurs, il faut des aristocrates : les patriotes ne s'en chargeraient pas ${ }^{77}$. D'autres agissent avec un zèle derrière lequel il faut percevoir de la crainte, assurant aux autorités municipales qu'ils ne défendront que les causes que ces mêmes autorités auront entérinées ${ }^{79}$. C'est surtout durant l'épisode de la Terreur que la méfiance envers les hommes de loi se cristallise. D'un département à l'autre, ceux qui ont fait de la défense en justice leur activité traversent forcément la période avec une certaine inquiétude. Lattachement aux valeurs ancestrales de la profession et la nécessité de défendre en toute circonstance laccusé donnent suffisamment de volonté aux hommes de loi pour poursuivre vaillamment leur ministère. La décennie révolutionnaire leur fait prendre conscience de la crainte qu'un défenseur trop libre peut inspirer à l'autorité politique. Lassaut mené à leur encontre n'est pas ultime. Au moment du rétablissement de l'Ordre des avocats en 1810, Napoléon saura s'en souvenir pour encadrer l'exercice de la profession dans des bornes raisonnables pour le gouvernement impérial.

En 1791, les rédacteurs de la Gazette des tribunaux soulignent précocement que l'absence des "membres distingués qui composoient l'ancien ordre des avocats [...] laisse un grand vide dans les nouveaux tribunaux ${ }^{80}$.

(75) Henri Wallon, Histoire du Tribunul révolutionnaire de Paris, Paris, 1882, t. 1, p. 465.

(76) E. LECESNe, Arrus sous la Révolution, Arras, 1883, t. 2, p. 161.

(77) H. Chauvot, Le harreau de Bordeaux de 1775 à 1815, Paris, 1856, p. 314.

(78) C. Lavaux, Les campagnes d'un avocat, Paris, 1815, p. 23.

(79) AD Vienne, L462.

(80) T. 2, p. 10. 
Toute la destinée de la défense officieuse est bel et bien là. L'erreur en partie assumée des Constituants a été de croire que la défense en justice pouvait échapper à ceux qui, jusqu'alors, en avaient fait leur métier. La fonction libre, débarrassée de règles professionnelles, ne signifie pas une défense sans barreaux ou, tout au moins, de ce qu'il en reste. La rupture s'est opérée au niveau institutionnel, avec une part d'utopie qui a conduit à l'échec du principe de la liberté de la défense et à son corollaire, la gratuité. En revanche, les hommes sont restés et ont contribué à donner une certaine crédibilité à la défense officieuse qui, à défaut d'avoir été un service d'ami, est restée un creuset où le justiciable a trouvé son conseil. Lexpérience a permis de mesurer à quel point défendre est un métier où l'amateur a peu de chances de réussir, indépendamment de tout point de vue politique. Le droit et la procédure restent complexes, surtout pour des particuliers dépourvus de connaissances juridiques. La parenthèse de la défense officieuse a fini par se refermer et les professionnels de la défense, praticiens de jadis, ont retrouvé un statut. Parmi eux, on a tout de même compté quelques rares amateurs qui n'étaient pas prédestinés, du fait de leur qualification initiale, à intégrer le barreau. Lexistence de ces exceptions montre que les espoirs de la Constituante n'étaient pas totalement illusoires.

Nicolas DERASSE

Université de Lille II Centre d'Histoire Judiciaire (UMR 8025)

1 , place Déliot 59000 Lille

nicolas.derasse@univ-lille2.fr 